\title{
Der Martingalansatz zur Auswertung klinischer Studien im Rahmen der Survival Analysis
}

\author{
Arnold Janssen
}

\begin{abstract}
Die Martingaltheorie liefert ein eindrucksvolles Beispiel für eine anspruchsvolle mathematische Theorie, deren Denkweise sich direkt in der Praxis durchgesetzt hat und großen Einfluss besitzt.

Neben den gefeierten Anwendungen in der Finanzmathematik (mit dem Nobelpreis für

Wirtschaftswissenschaften 1997) wird in den Lebenswissenschaften ausgehend von Martingalmethoden für Zählprozesse der Bogen bis hin zur Auswertung klinischer Studien in der Medizin gespannt. Grundlegend sind dabei die Arbeiten von Kaplan und Meier (1958) und Cox (1972), die die Rangliste der am häufigsten zitierten Arbeiten mit einem mathematischen Inhalt anführen. Für seine Beiträge zur Statistik und insbesondere zur Survival Analysis erhält Sir David Cox (Oxford) den erstmals vergebenen „International Prize in Statistics“.
\end{abstract}

In der zweiten Hälfte des 20. Jahrhunderts wurden Martingalmethoden als wesentlicher Beitrag zur Wahrscheinlichkeitstheorie entwickelt. Zunächst stellte sich heraus, dass diese wunderbaren Instrumente die mathematische Theorie und insbesondere die Theorie stochastischer Prozesse weiterentwickeln konnten. Martingale $\left(M_{t}\right)_{t \geq 0}$ bestehen aus integrierbaren reellen Zufallsvariablen, die eine Interpretation als akkumulierte Auszahlungen für faire Spiele besitzen. Wird die bis zur Zeit $t$ verfügbare Information in einer $\sigma$-Algebra $\mathcal{F}_{t}$ gesammelt, so ist die Martingaleigenschaft durch die Gleichung

$$
E\left(M_{t} \mid \mathcal{F}_{s}\right)=M_{s}
$$

für die bedingte Erwartung von $M_{t}$ unter $\mathcal{F}_{s}$ für alle $s \leq t$ gegeben.

In Verbindung mit der parallel entwickelten stochastischen Analysis bilden Martingale die Grundlage für die moderne Finanzmathematik und erfahren hier eine breite praktische Anwendung, siehe z. B. die frühen Arbeiten von Black und Scholes (1973) und Merton (1971), die 1997 in der Vergabe des Nobelpreises für Wirtschaftswissenschaften an Myron Scholes and Robert Merton mündeten. Eine Würdigung ist in dem Beitrag von Föllmer (1998) in den DMV Mitteilungen zu finden.

Daneben haben die Martingalmethoden Eingang in die moderne biometrische Forschung gefunden und zu einer großartigen Erfolgsgeschichte beigetragen. Die Anwendung dieser tiefliegenden mathematischen Theorie reicht bis in die tägliche klinische Arbeit. Über diese wichtige auf Martingalen beruhende Forschungsrichtung soll hier berichtet werden.

Schon im 19. Jahrhundert wurden in der Versicherungsmathematik Sterbetafeln verwendet, die über empirische
Ausfallraten (2) eine dynamische Anpassung von Überlebensdaten und deren Verteilungen ermöglichen. Im Folgenden bezeichne $T$ eine diskrete Zufallsvariable mit Werten in $\mathbb{N}$, die eine Lebenszeit (oder Zuverlässigkeit) modelliert. Ist $p_{m}=P(T=m)$ die Wahrscheinlichkeit für einen Ausfall zum Zeitpunkt $m$, so bezeichnet

$$
r_{m}:=\frac{p_{m}}{P(T \geq m)}=P(T=m \mid T \geq m)
$$

die Ausfallsrate, d.h. die bedingte Wahrscheinlichkeit in $m$ auszufallen unter der Bedingung des Erlebens des Zeitpunkts $m$, sofern das Ereignis $\{T \geq m\}$ positive Wahrscheinlichkeit besitzt. Aus gegebenen Raten $\left(r_{m}\right)_{m}$ lässt sich leicht die Verteilung von $T$ durch die Produktformel

$$
P(T>k)=\sum_{n=k+1}^{\infty} p_{n}=\prod_{1 \leq m \leq k}\left(1-r_{m}\right), k \in \mathbb{N}
$$

für die Survivalfunktion $k \mapsto P(T>k)$ zurückgewinnen. Für stetige Verteilungen mit einer Lebesgue-Dichte $f$ auf $\mathbb{R}_{+}$ ist eine analoge Begriffsbildung durch die Hazardrate $\lambda(\cdot)$ gegeben,

$$
\lambda(t)=\frac{f(t)}{P(T \geq t)}, t>0,
$$

die als altersbedingte Ausfallsrate bezeichnet wird. Ist $f$ stetig an der Stelle $t$, so gilt für hinreichend kleine positive $\varepsilon>0$

$$
P(T \in[t, t+\varepsilon] \mid T \geq t) \approx \varepsilon \lambda(t) .
$$

Die Wahrscheinlichkeit eines Ausfalls im Intervall $[t, t+\varepsilon]$ ist unter der Hypothese $\{T \geq t\}$ approximativ $\varepsilon \lambda(t)$. Die 


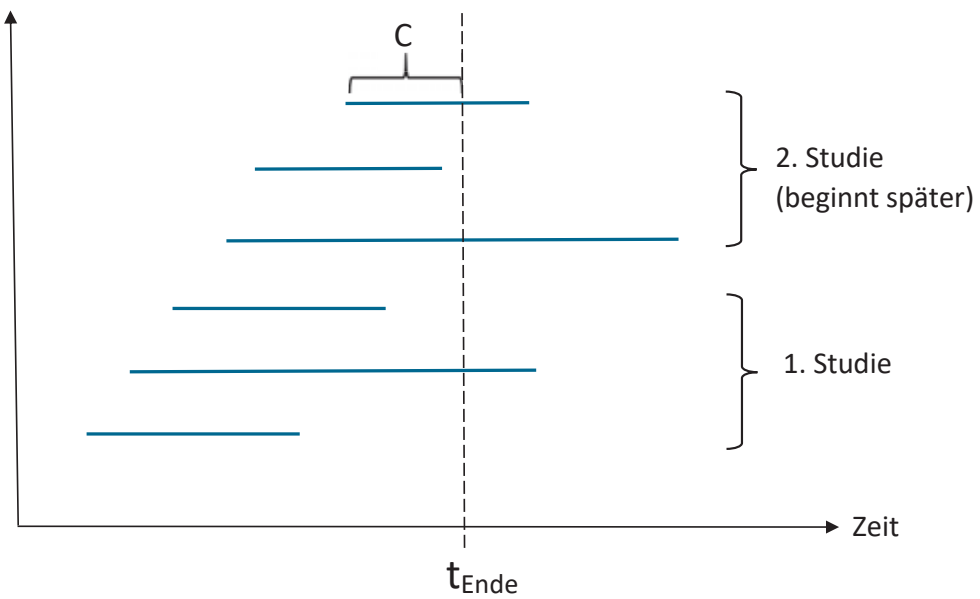

Grafik 1. Überlebenszeiten $T$ (schwarze Linien) und potenzielle Zensierungsvariablen $C$

Survivalfunktion berechnet sich hier als

$$
P(T>t)=\exp \left(-\int_{0}^{t} \lambda(u) d u\right)
$$

aus der Hazardrate, sofern die linke Seite positiv ist.

Seit langer Zeit wird in der Versicherungsmathematik mit diesen Modellen und analogen empirischen Größen erfolgreich gearbeitet. An dieser Stelle treten bereits Martingale auf. Es seien $T_{1}, \ldots, T_{n}$ unabhängig identisch verteilte Zufallsvariablen (Überlebenszeiten) mit Werten in $\mathbb{N}$. Die erzeugte $\sigma$-Algebra

$$
\mathcal{F}_{k}=\sigma\left(\mathbb{I}_{[0, r]}\left(T_{i}\right): 0 \leq r \leq k, 1 \leq i \leq n\right),
$$

gegeben durch die Indikatorfunktion $\mathbb{I}_{[0, r]}$ des Intervalls $[0, r]$, enthält die vorliegende Information bis zur Zeit $k$. Wird $k$ als das $k$-te Lebensjahr einer homogenen Population interpretiert, so haben zu Beginn der $k$-ten Periode noch

$$
Y_{k}=\sum_{j=1}^{n} \mathbb{I}_{[k, \infty)}\left(T_{j}\right)
$$

Probanden überlebt. Der Mediziner sagt: $Y_{k}$ Personen sind noch unter Risiko.

Der Zählprozess der Ausfälle

$$
N_{k}=\sum_{j=1}^{n} \mathbb{I}_{[0, k]}\left(T_{j}\right)
$$

besitzt als Zuwächse

$$
N_{k}-N_{k-1}=\sum_{j=1}^{n} \mathbb{I}_{\{k\}}\left(T_{j}\right)
$$

die Zahl der Ausfälle zur Zeit $k$. Zu Beginn der $k$-ten Periode werden unter Kenntnis von $\mathcal{F}_{k-1}$

$$
\mathbb{E}\left(N_{k}-N_{k-1} \mid \mathcal{F}_{k-1}\right)=r_{k} Y_{k}
$$

Ausfälle erwartet, wenn $r_{k}$ die gemeinsame Ausfallrate (2) bezeichnet. Standardargumente liefern jetzt die DoobMeyer-Zerlegung des Zählprozesses

$$
k \mapsto N_{k}=M_{k}+A_{k}
$$

in einen Martingalteil $\left(M_{k}\right)_{k}$ und einen $\mathcal{F}_{k-1}$-messbaren Kompensator

$$
A_{k}:=\sum_{j=1}^{k} \mathbb{E}\left(N_{j}-N_{j-1} \mid \mathcal{F}_{j-1}\right)=\sum_{j=1}^{k} r_{j} Y_{j}
$$

durch Zentrieren der Zuwächse $N_{k}-N_{k-1}$ am bedingten Erwartungswert (9). Die Versicherer können für große homogene Populationen und bei einer sehr guten Datenlage meistens gut mit diesen Ansätzen arbeiten. Zudem werden hier durch spezielle Verteilungsannahmen gute Modellapproximationen erreicht. In der Technik können analog häufig Haltbarkeitsprobleme mit Weibull-Verteilungen parametrisch modelliert werden. Die Variablen $T_{i}$ beschreiben im Allgemeinen nicht nur Todeszeitpunkte, sondern sogenannte Event-Zeiten bis zum Eintreten eines relevanten Ereignisses. In den Wirtschaftswissenschaften kann $T_{i}$ z. B. die Dauer einer Arbeitslosigkeit angeben.

In der Medizin ist die Datenlage in der Regel nicht so komfortabel. An der Tagesordnung sind geringe Fallzahlen und unterschiedliche Merkmale der Probanden (Alter, Geschlecht, ...), die in Kovariablen zusammengefasst werden. Besonders schwerwiegend ist die partiell fehlende Information, die in der Praxis an der Tagesordnung ist und sich auch bei sorgfältiger Planung nicht vermeiden lässt. Die Überlebenszeiten $T_{i}$ können durch äußere Einflüsse oft nicht 


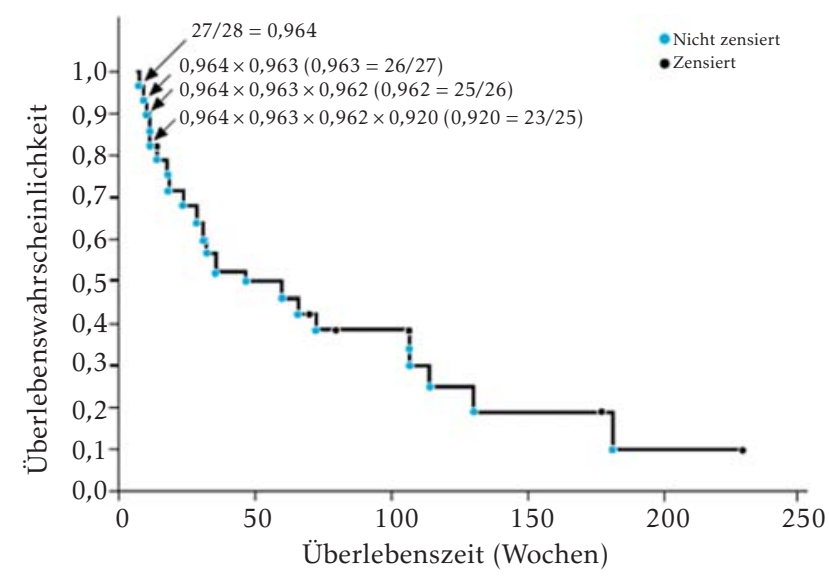

Grafik 2. Kaplan-Meier-Schätzer für die Überlebenszeit von 28 Zungenkrebspatienten. Es wird die Wahrscheinlichkeit dargestellt, dass ein Patient eine Zeit (in Wochen) überlebt.

vollständig beobachtet werden. Zur Illustration dient die Grafik 1.

Die x-Achse beschreibt die Kalenderzeit mit jeweils drei zu unterschiedlichen Zeiten beginnenden Überlebenszeiten in einem Zweistichprobenproblem, bei dem z. B. zwei medizinische Therapien verglichen werden sollen. Typischerweise sind nicht alle $T_{i}$ vollständig beobachtbar, sondern nur bis zum Ende der Studie bis zur Zeit $t_{\text {Ende. In dem Beispiel }}$ liegen drei von sechs Lebenszeiten zum Auswertungszeitpunkt $t_{\text {Ende }}$ noch nicht vor. Aber gerade diese „guten Risiken" enthalten wertvolle Informationen und müssen bei der Datenauswertung unbedingt berücksichtigt werden. Dazu dient das Modell der sogenannten zufällig rechtszensierten Variablen, das wie folgt motiviert werden kann. Ist $t_{i}$ die Kalendereintrittszeit des $i$-ten Probanden, so ist $C_{i}:=t_{\text {Ende }}-t_{i}$ die maximale Beobachtungsspanne in der Studie, siehe Grafik 1. Angenommen wird jetzt, dass der Eintrittszeitpunkt $t_{i}$ unabhängig von der potenziellen Überlebenszeit ist, d.h. $T_{i}$ und $C_{i}$ sind unabhängig. Die Variable $C_{i}$ wird als zufällige Zensierungsvariable bezeichnet. Demnach liegt das Modell

$$
\left(X_{i}, \Delta_{i}\right):=\left(\min \left(T_{i}, C_{i}\right), \mathbb{I}\left(T_{i} \leq C_{i}\right)\right), 1 \leq i \leq n,
$$

für die beobachtbare Datenstruktur vor, wobei die Indikatorfunktion $\Delta_{i}=\mathbb{I}\left(T_{i} \leq C_{i}\right)$ den Zensierungsstatus angibt. Die Werte mit $\Delta_{i}=0$ und $X_{i}=C_{i}$ heißen zensiert (bzw. unzensiert, wenn $X_{i}=T_{i}$ und $\Delta_{i}=1$ gilt).

Das Modell (12) erlaubt eine viel umfassendere Interpretation nicht nur als Zensierung durch ein Studienende. Vielmehr kann $C_{i}$ jede beobachtbare Zensierungszeit angeben, die unvorhergesehen oder kontrolliert zu einem Datenverlust führt, solange dieser unabhängig von der potenziellen Verlustzeit $T_{i}$ ist. Auch in den Wirtschaftswissenschaften spielen zensierte Daten eine Rolle. Die Dauer einer Arbeitslosigkeit kann zensiert sein, wenn zum Auswertungszeitpunkt noch keine Neueinstellung erfolgt ist. Analog zu (7) und (8) werden für das zensierte Modell (12) die Zählprozesse $N(\cdot)$ der wahren Ausfälle

$$
N(t):=\sum_{j=1}^{n} \Delta_{j} \mathbb{I}_{[0, t]}\left(X_{j}\right), \quad Y(t):=\sum_{i=1}^{n} \mathbb{I}_{[t, \infty]}\left(X_{i}\right)
$$

und der Personen $Y(t)$ unter Risiko zur Zeit $t$ eingeführt. $\mathrm{Zu}$ einer homogenen Population mit unabhängig identisch verteilten Überlebenszeiten schätzt

$$
\hat{r}(t)=\frac{N(t)-N(t-)}{Y(t)}
$$

die über $\left(X_{i}, \Delta_{i}\right)$ zugängliche Ausfallrate der $T_{i}$ zur Zeit $t$. Der Zähler hängt dabei nur von unzensierten Daten ab. Dabei wird $t$ nicht nur als Variable auf dem Gitter $\mathbb{N} \cup\{0\}$ verstanden, sondern kann Werte in den positiven Zahlen annehmen. Allgemeiner als in (3) ergibt sich durch Einsetzen in die Produktformel der berühmte Kaplan-Meier-Schätzer

$$
\hat{S}(t)=\prod_{0 \leq s \leq t}(1-\hat{r}(s))
$$

als Schätzer der Survivalfunktion $S(t)=P\left(T_{i}>t\right)$, auch wenn die $T_{i}$ nicht immer alle direkt beobachtbar sind. Die Grafik 2 (siehe Ziegler (2014)) gibt beispielhaft den Verlauf des Kaplan-Meier-Schätzers für Krebspatienten einer Studie an.

Der Kaplan-Meier-Schätzer ist der nichtparametrische Maximum-Likelihood-Schätzer für die Survivalfunktion $S(\cdot)$. Der Schätzer ist das Standardwerkzeug der medizinischen Statistik und allgegenwärtig in medizinischen Publikationen. Bis Ende 2016 wurde die Arbeit von Kaplan und Meier (1958) mehr als 48 500-mal zitiert, eine astronomische Zahl von Zitaten für eine Arbeit mit mathematischen Inhalten. Kaplan und Meier bedanken sich in der Arbeit bei dem bekannten Statistiker John W. Tukey (Princeton), der auch durch seine Beiträge zur Topologie bekannt wurde. 
Die mathematische Analyse des Kaplan-Meier-Schätzers ist sehr anspruchsvoll und beruht auf ausgefeilten Martingalargumenten. Erst 1982 gelang Jon A. Wellner (20162017 Präsident des Institute of Mathematical Statistics) der Beweis der asymptotischen Optimalität des Kaplan-MeierSchätzers für das volle nichtparametrische Zensierungsmodell. Noch später gaben Stute und Wang (1993) einen ersten vollständigen Beweis für die starke Konsistenz des Kaplan-Meier-Schätzers für den größtmöglichen Zeitbereich an. Dabei wird der Nachweis der fast sicheren Konvergenz der Schätzer mit inversen Martingalen geführt. Im Anhang wird an einem einfachen Beispiel die Konstruktion des Kaplan-Meier-Schätzers demonstriert, die für jeden Anwender zugänglich ist. Die Schätzung von multivariaten Survivalfunktionen ist für zensierte Daten sehr schwierig und nach wie vor ein Forschungsthema der mathematischen Statistik.

Ein weiterer Meilenstein sind die Arbeiten von Sir David R. Cox (Oxford) zur Survivalanalysis für Zensierungsmodelle. Für seine Beiträge zur Statistik, insbesondere zur Analyse von Überlebensdaten, erhält der 92-jährige Sir Cox den International Prize in Statistics, der 2017 erstmals gemeinsam von fünf bedeutenden Fachgesellschaften vergeben wird. Dies sind The American Statistical Association mit 19 ooo Mitgliedern, The Institute of Mathematical Statistics (450o Mitglieder), The Royal Statistical Society (70oo Mitglieder), The International Biometric Society und The International Statistical Institute. Das sogenannte Cox-Modell für zensierte Daten (16) von David R. Cox (1972) wurde bis Ende 2016 bereits $42600-m a l$ zitiert und ergänzt in idealer Weise die Kaplan-Meier-Theorie. In dem Modell wird die in (4) eingeführte Hazardrate $\lambda(t, z)$ für einen Probanden mit den individuellen Ausprägungen $\left(z_{1}, \ldots, z_{p}\right) \in \mathbb{R}^{p}$ (etwa Alter, Geschlecht, ...) zur Zeit $t$ durch das Regressionsmodell

$$
\lambda(t, z)=\exp \left(z_{1} \beta_{1}+\ldots+z_{p} \beta_{p}\right) \lambda_{0}(t), t>0,
$$

dargestellt. Dabei sind $\beta_{1}, \ldots, \beta_{p}$ unbekannte schätzbare reelle Einflussgrößen. Ferner ist $\lambda_{0}(\cdot)$ eine vollständig unbekannte nichtparametrische sogenannte „baseline“ Hazardrate, die als Gestaltsfunktion angesehen wird. Der Charme des Ansatzes besteht darin, dass das relative Ausfallrisiko

$$
\frac{\lambda(t, z)}{\lambda(t, \tilde{z})}=\exp \left(\left(z_{1}-\tilde{z}_{1}\right) \beta_{1}+\ldots+\left(z_{p}-\tilde{z}_{p}\right) \beta_{p}\right)
$$

für zwei Probanden mit Ausprägungen $z$ und $\tilde{z}$ der Kovariablen nicht von der Verteilungsstruktur abhängt, die durch den unendlich dimensionalen Parameter $\lambda_{0}$ gegeben ist. Ein Mediziner versteht das Cox-Modell sehr gut. Beispielsweise kann ein Raucher ein um einen Faktor (17) erhöhtes Krankheitsrisiko besitzen. Der Anwender braucht dabei die durch $\lambda_{0}$ gegebene Verteilung nicht zu modellieren! In der Praxis ist dies ein großer Vorteil, da häufig gängige parametrische Lebenszeitmodelle wegen zu geringer Fallzahlen und fehlender Information durch die zusätzlich vorhandene Zensierung nicht angepasst werden können.

Modelle vom Typ Cox und deren Verallgemeinerungen mit zeitabhängigen relativen Ausfallrisiken haben sehr bald Eingang in die klinische Forschung gefunden und sind heu- te fester Bestandteil statistischer Softwarepakete. Insbesondere wurde die Schätz- und Testtheorie für die unbekannten Parameter entwickelt. Die mathematische Behandlung erfolgt wiederum mit Martingalmethoden. Die Weiterentwicklung der Survivalanalysis nach der Arbeit von Cox (1972) wird von einer stürmischen Entwicklung der mathematischen Statistik getragen. Die Arbeit von Cox hat Ood Aalen (Oslo) inspiriert, der in der Publikation von 1978 Martingalund Zählprozessargumente in die Theorie einbrachte. Die Arbeit entstammt der Dissertation von Aalen in Berkeley, die auch von Le Cam (Berkeley) angeregt wurde. Richard Gill (jetzt Leiden) hat 1980 die Arbeit von Aalen aufgegriffen und die Theorie zusammen mit seinen Koautoren vervollständigt und in einem umfassenden Werk zur Blüte und zur anschließenden Praxisreife geführt, siehe die Monografie von Andersen, Borgan, Gill und Keiding (1993). Für weitere Referenzen zum Thema Kaplan-Meier-Schätzer sind die Handbücher Handbook of Statistics, Vol. 23, und Handbook of survival analysis von Klein, van Houwelingen, Ibrahim and Scheike (2014) empfehlenswert.

Die bisherigen Ausführungen beziehen sich auf die Modelle der Survivalanalysis und auf die Diskussion von Schätzern. Für den Praktiker sind natürlich auch Tests für die Mehrstichprobenprobleme von großer Bedeutung, wenn - wie z. B. in der Grafik 1 dargestellt - verschiedene Studien beurteilt und verglichen werden. Die Testtheorie ist ebenfalls in Andersen, Borgan, Gill und Keiding (1993) dargestellt, siehe auch den Übersichtsartikel von Janssen und Werft (2004). Neuere Ergebnisse der Düsseldorfer Arbeitsgruppe über Tests für Survivaldaten sind z. B. in Brendel, Janssen, Mayer und Pauly (2014) zu finden.

Die Arbeit von Cox hat ferner einen weiteren bedeutenden Zweig der Statistik angeregt, die Analyse allgemeiner semiparametrischer Modelle. Wie in (16) sind semiparametrische Modelle durch einen endlich dimensionalen interessierenden Modellparameter $\left(\beta_{1}, \ldots, \beta_{p}\right)$ und einen unendlich dimensionalen Neben- und Störparameter gegeben, der in (16) durch die baseline Hazardrate $\lambda_{0}(\cdot)$ beschrieben wird. Diese Theorie ist in der Monografie von Bickel, Klaassen, Ritov und Wellner (1993) enthalten.

Die Arbeiten von Kaplan und Meier (1958) und Cox (1972) können als Durchbruch in der Statistik bezeichnet werden, siehe dazu die Ansätze in der Serie Breakthroughs in Statistics (1992), Ed. Kotz und Johnson. Die verwendeten Martingalmethoden wirken bis in die aktuellen klinischen Studien nach und gehen damit eine bedeutende Symbiose zwischen der Mathematik und praktischen Anwendungen ein.

\section{Anhang}

Im Folgenden soll anhand eines kleinen Beispiels erklärt werden, wie der Kaplan-Meier-Schätzer konstruiert wird.

Nehmen wir an, dass $n=5$ Realisationen von $\left(X_{i}, \Delta_{i}\right)$ (12) durch die Werte

$$
(3,1),(1,1),(5,1),(4,0),(2,0)
$$

mit unzensierten Werten $x=3$, 1 und 5 und zensierten 

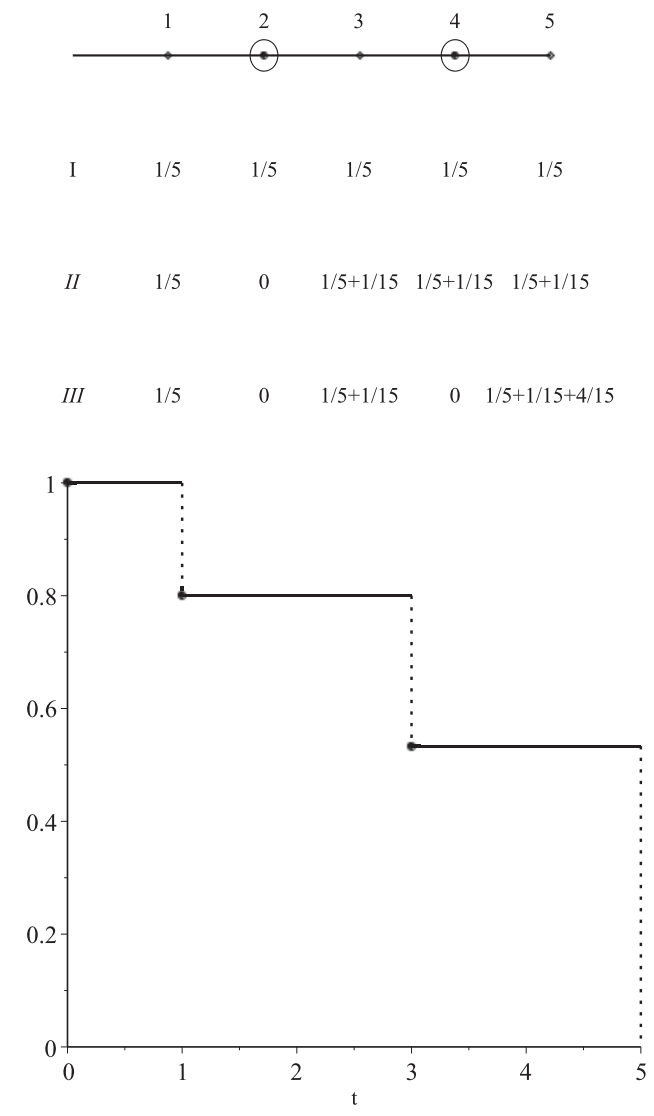

Grafik 3. Daten und ihre Bewertung: Konstruktion des Kaplan-Meier-Schätzers $t \mapsto \hat{S}(t)$. Die empirischen Gewichte $(14)$ sind $\hat{r}(1)=\frac{1}{5}, \hat{r}(2)=0 . \hat{r}(3)=$ $\frac{1}{3}, \hat{r}(4)=0, \hat{r}(5)=1$.

Punkten $x=4$ und 2 gegeben sind. Zunächst werden die $x$-Werte der Größe nach in Grafik 3 aufgetragen und die zensierten Werte $(2,0)$ und $(4,0)$ werden durch einen Kreis markiert. Das Schema wird jetzt mit fortschreitender Zeit von links nach rechts wie folgt abgearbeitet, siehe Grafik 3 .

Im ersten Schritt (I) werden alle Punkte 1, ., 5 mit dem empirischen Gewicht $\frac{1}{5}$ belegt. Ausgehend vom kleinsten Wert wird jetzt das erste zensierte Datum ermittelt, hier $(2,0)$. Das Gewicht dieses Punktes wird danach gleichmäßig auf alle nachfolgenden Beobachtungspunkte verteilt, siehe Schritt II. Hier wird allen Punkten 3-5 der Wert $\frac{1}{15}$ zusätzlich zugeschlagen und das Gewicht vom Punkt 2 wird auf 0 gesetzt. Dieses Verfahren wird fortgesetzt und das nächste zensierte Datum (hier $(4,0)$ ) wird gesucht. Wiederum wird das vorhandene Gewicht an die Nachfolger verteilt.

In dem Beispiel wird in Schritt III eine Verteilung erreicht, die als Survivalfunktion den Kaplan-Meier-Schätzer besitzt, siehe die unten stehende Grafik. Ist das größte Datum zensiert, so erhält der größte Wert das Gewicht 0 und die Gewichte addieren sich insgesamt nicht mehr auf 1. In der Praxis können solche Effekte auftreten.

\section{Literatur}

[1] Aalen, O. (1978), Nonparametric inference for a family of counting processes, Ann. Statist., 6, 701-726.

[2] Andersen, P. K., Borgan, O., Gill, R. D., und Keiding, N. (1993), Statistical models based on counting processes, Springer, New York.

[3] Balakrishnan, N., und Rao, C. R., (2004), Advances in Survival Analysis, Handbook of Statistics, Vol. 23, Elsevier North-Holland.

[4] Brendel, M., Janssen, A., Mayer, C.-D. und Pauly, M. (2014), Weighted logrank permutation tests for randomly right censored life science data. Scandinavian Journal of Statistics 41, 742-761.

[5] Bickel, P. J., Klaassen, C. A. J., Ritov, Y. und Wellner, J. A. (1998), Efficient and Adaptive Estimation for Semiparametric Models. John Hopkins Series in the Mathematical Sciences.

[6] Black, F. und Scholes, M., (1973), The pricing of options and corporate liabilities. The Journal of Political Economy, vol. 81, 637-654.

[7] Cox, D. R. (1972), Regression models and life-tables. Journal of the Royal Statistical Society. Series B, Vol. 34, 187-220.

[8] Föllmer, H., (1998), Ein Nobel-Preis für Mathematik? Mitteilungen der DMV 1/98, 4-7.

[9] Janssen, A. und Werft, W. (2004), A survey about the efficiency of two-sample survival tests for randomly censored data. Mitteilungen aus dem Mathematischen Seminar Giessen, Heft 254, $1-47$. 
[10] Gill, R. D. (1980), Censoring and Stochastic Integrals. Math. Centre Tracts 124, Matematisch Centrum Amsterdam.

[11] Kaplan, E. L. und Meier, P. (1958), Nonparametric estimation from incomplete observations. JASA 53 (282): 457-481.

[12] Klein, J. P., van Houwelingen, H. C., Ibrahim, J. G., Scheike, T. H. (2014), Handbook of survival analysis. Chapman and Hall CRC handbooks of modern statistical methods.

[13] Kotz, S. und Johnson, N. L. (1992), Breakthroughs in Statistics, Springer Series in Statistics.
[14] Merton R. (1971), Optimum consumption and portfolio rules in a continuous-time model. Journal of Economic Theory, vol. 3, $373-413$.

[15] Stute, W. und Wang, J.-L. (1993), The strong law under random censorship. Ann. Statist., 21, 1591-1607.

[16] Wellner, Jon A. (1982), Asymptotic optimality of the product limit estimator. Ann. Statist. 10, 595-602.

[17] Ziegler A., Lange S. und Bender R. (2014), Überlebenszeitanalyse: Eigenschaften und Kaplan-Meier Methode. Artikel Nr. 15 der Statistik-Serie in der DMW.

Univ.-Prof. Dr. Arnold Janssen, Mathematisches Institut,

Heinrich-Heine-Universität Düsseldorf, Universitätsstraße 1, 40225 Düsseldorf janssena@math.uni-duesseldorf.de

Arnold Janssen hat den Lehrstuhl für Mathematische Statistik und Wahrscheinlichkeitstheorie am Mathematischen Institut der Heinrich-Heine Universität Düsseldorf inne. Seine

Hauptarbeitsgebiete sind die asymptotische und nichtparametrische Statistik mit

Anwendungen in den Lebenswissenschaften sowie die Analyse hochdimensionaler Daten.

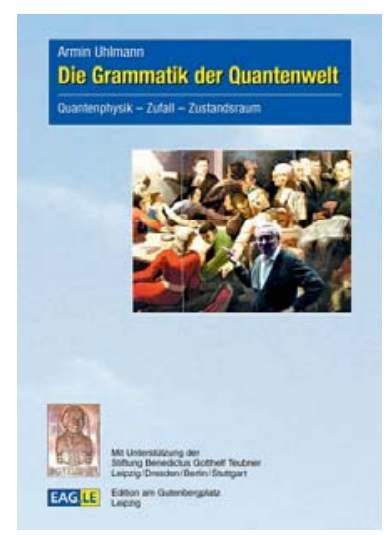

A. Uhlmann. EAGLE 096.

1. A. 2017. 978-3-95922-096-5

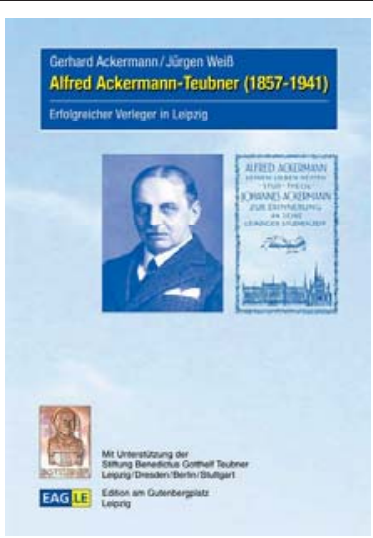

G. Ackermann / J. Weiß. EAGLE 090.

1. A. 2016. 978-3-95922-090-3

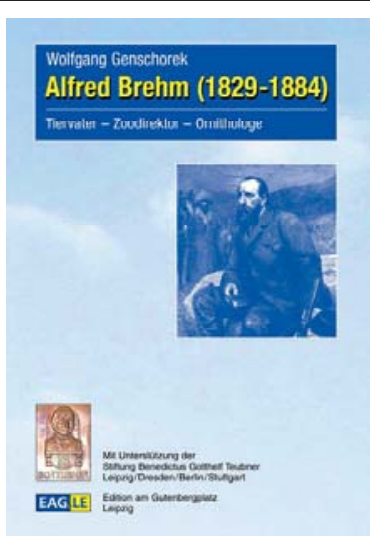

W. Genschorek. EAGLE 092.

1. A. 2016. 978-3-95922-092-7
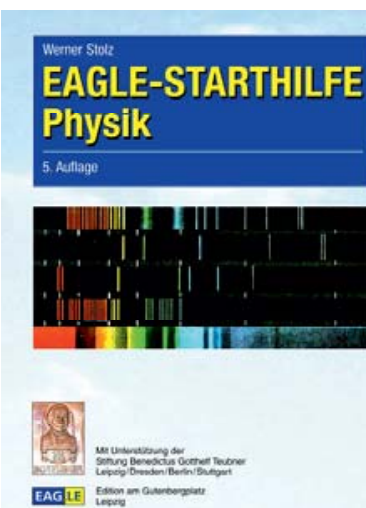

W. Stolz. EAGLE 086

5. A. 2015. 978-3-95922-086-6

Edition am Gutenbergplatz Leipzig: www.eagle-leipzig.de I www.eagle-leipzig.de/starthilfen.htm I https:/ltwitter.com/EagleLeipzig NEU/März 2017: Michael Börngen (Leipzig), Heinrich Wilhelm Brandes (1777-1834). Erfinder der Wetterkarte, Leipzig 1817/1826. NEU/Februar 2017: Armin Uhlmann (Leipzig), Die Grammatik der Quantenwelt. Quantenphysik - Zufall - Zustandsraum. EAGLE 2017

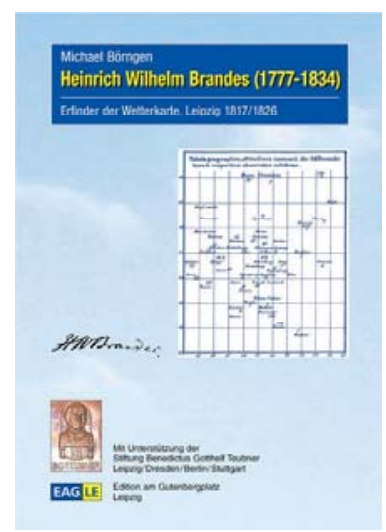

M. Börngen. EAGLE 085.

1. A. 2017. $978-3-95922-085-9$

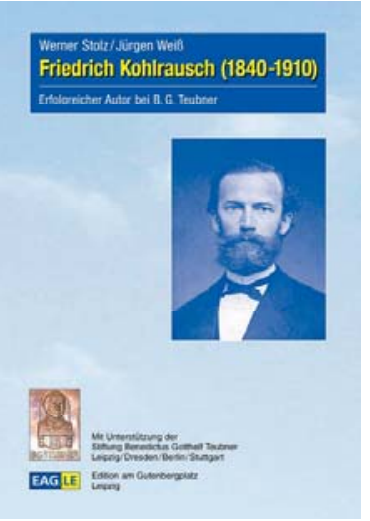

W. Stolz / J. Weiß. EAGLE 095. 1. A. 2017. 978-3-95922-095-8

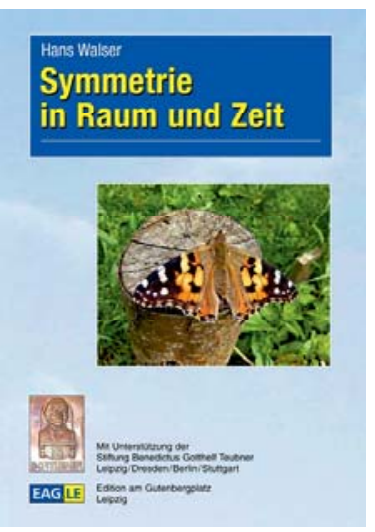

H. Walser. EAGLE 046.

1. A. 2014. 978-3-937219-46-2

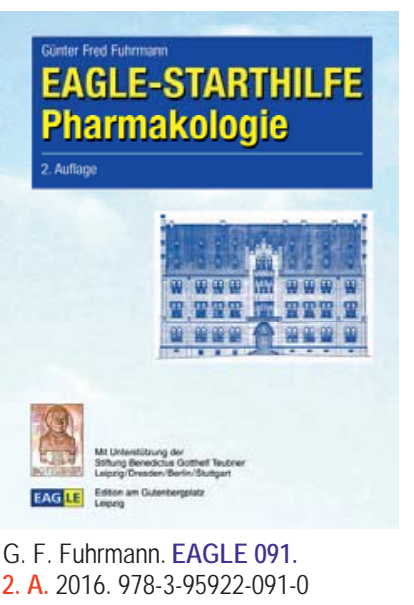

